\title{
Volume of Distribution for Dosing Interval by Fraction Normalized by Dose
}

National Cancer Institute

\section{Source}

National Cancer Institute. Volume of Distribution for Dosing Interval by Fraction

Normalized by Dose. NCI Thesaurus. Code C111366.

The volume of distribution associated with the terminal slope following extravascular administration divided by the fraction of dose absorbed, calculated using AUCTAU, divided by the dose. 